Oral presentation

Open Access

\title{
Novel mechanisms of anti-hypertrophic effects from PDE5A inhibition
}

\author{
David A Kass*, Norimichi Nagayama, Manling Zhang, Dong-ik Lee, \\ Steven Hsu, Elizabeth Ketner and Eiki Takimoto
}

Address: Division of Cardiology, Department of Medicine, and Department of Biomedical Engineering, The Johns Hopkins Medical Institutions, Baltimore, Maryland USA

Email: David A Kass* - dkass@jhmi.edu

* Corresponding author

from 4th International Conference of cGMP Generators, Effectors and Therapeutic Implications

Regensburg, Germany. 19-21 June 2009

Published: II August 2009

BMC Pharmacology 2009, 9(SuppI I):SI8 doi:10.1I86/I47I-2210-9-SI-SI8

This abstract is available from: http://www.biomedcentral.com/I47I-22I0/9/SI/SI8

(C) 2009 Kass et al; licensee BioMed Central Ltd.

\section{Background}

Phosphodiesterase type-5 is a cGMP-selective PDE expressed in heart, and growing evidence supports its importance in modifying cardiac stress response signalling. Recent human studies have reported PDE5A myocyte expression notably in disease conditions such as hypertrophy and dilated heart failure. We have shown that upregulating PDE5A in isolated myocytes stimulates cellular hypertrophy. This is now demonstrated in vivo using a new tetracycline-controllable myocyte-targeted overexpression mouse model. Upregulation of PDE5A in myocytes lowers PKG activation, and results in gradual chamber dilation, hypertrophy, and dysfunction. In hearts from younger transgenic animals (without basal abnormalities), PDE5A overexpression worsens the response to pressure-overload stress. A major target of PDE5A-inhibitor mediated anti-hypertrophic signalling appears to be Gaq-receptor activation by both neurohumoral ligands and mechanical stimuli. Here we report several new mechanisms by which this is achieved.

\section{Results}

In one study, mice lacking the regulator of G-protein signalling 2 (RGS2) show profoundly worse responses to pressure-overload, with early hypertrophy, dilation, dysfunction, and lethality. Despite elevation of PKG in these hearts treated with sildenafil as in littermate controls, the RGS2-null heart does not respond to PDE5A inhibition (with less hypertrophy, improved function, etc.) whereas the controls do. PKG-RGS2 interaction is important to this signalling. In a second series of studies, we identified a novel PKG regulation of transient receptor potential canonical channel protein (TRPC6). TRPC6 has been shown to serve as a ligand and stretch responsive channel for Ca2+ conductance - that couples to Ca2+-calmodulin/ calcineurin/NFAT activation. Recent evidence in embryonic aortic smooth muscle has shown TRPC6 can be PKG phosphorylated at $\mathrm{T} 11$, reducing conductance. We have found PKG also phosphorylates TRPC6 in myocytes, identifying two functional sites, and that this is coupled with changes in conductance modulation by cGMP/PKG and with antihypertrophic signalling effects of sildenafil linked to the suppression of NFAT activity induced by Gqreceptor agonists. Gain or loss of function phosphormutants confirm the functionality of this modulation by PKG.

\section{Conclusion}

As TRPC6 is also thought to play an important role in hypertension, and gain of function missense mutations lead to human glomerulosclerosis, the ability of sildenafil to suppress conductance may have broader implications beyond cardiac disease. 